\title{
Laboratory values in a Spanish population of older adults: A comparison with reference values from younger adults
}

\author{
José Carlos Millán-Calenti, Alba Sánchez, Laura Lorenzo-López, Ana Maseda \\ Gerontology Research Group, Department of Medicine, University of A Coruña, Faculty of Health Sciences, Campus de Oza, \\ E-15006 A Coruña, Spain
}

\begin{abstract}
Objective: To examine the laboratory indices in a population aged 65 years or more and compare them with the reference values used for young adults. Study design: Distribution patterns of frequently used biochemical and hematological indices were examined in a sample $(\mathrm{N}=600)$ of non-institutionalized adults aged over 65 .

Outcome measures: The obtained values were compared with the reference intervals for young adults.

Results: On some of the indices analyzed, large proportions of the participants had values above the upper limit of the reference interval: glucose, $25.0 \%$; urea, 26.6\%; creatinine, $27.2 \%$ of males; total cholesterol, $54.6 \%$; and lowdensity lipoprotein cholesterol, 35.8\%. Of the participants who met the World Health Organization's diagnostic criteria for diabetes, $31.8 \%$ said they had not been previously diagnosed. Similarly, $74.9 \%$ of subjects with total cholesterol values above the reference value indicated in the European guidelines on cardiovascular disease said they had not been diagnosed with dyslipidemia, as did $75.5 \%$ of those with low-density lipoprotein cholesterol values above the European reference value. The proportion of participants who were not aware that they might be suffering from those disorders was significantly higher among those who reported not having visited their doctor within the last 6 months.

Conclusion: Further studies should examine whether the use of adapted, more appropriate reference

values for elderly populations will help physicians to make early and correct diagnoses and to decide

when medical intervention is required.

Keywords: Clinical chemistry variables, Reference values, Elderly
\end{abstract}

\section{Introduction}

Laboratory tests are used to detect pathology and confirm diagnoses. To interpret the results of these tests, it is necessary to compare them with reference values drawn from a healthy population. Unfortunately, the reference population often largely comprises young adults, and this may make the reference values inappropriate for an elderly patient. For example, significant agerelated changes have been observed in hematological profile [1]. Since the use of inappropriate reference values may impede the detection of pathologies in older adults, it would be useful to establish age-specific reference values.

Few papers have included reference values for adults aged over 65 and some even of these studies have included younger subjects [1,2]. A further problem is that where age-appropriate reference intervals have indeed been based wholly on samples of healthy older subjects [2-6], the criteria used to determine their 'healthy' status have varied across studies.

Most research done with elderly adults not suffering severe disorders has shown that their biochemical parameters are in fact within the conventional reference values for young adults [3,4]. Nevertheless, some biochemical and hematological indices have shown wider normal (healthy) reference intervals for older adults than for young adults [7].

A likely reason for the lack of reference values specific to an elderly population is that older adults have a relatively high prevalence of chronic pathologies such as diabetes, dyslipidemia, dementia, renal disease and anemia [8-11], as well as high comorbidity [12], which makes it difficult to find an appropriate healthy reference sample. Furthermore, a large proportion of older subjects regularly take medication [13] and many of them are dependent in activities of daily living (ADL) and instrumental activities of daily living (IADL) [14]. Again, the use of a strict health criterion is likely to result in a small and unrepresentative sample of the elderly population [5].

In this study we determined biochemical and hematological indices for a representative sample of adults aged over 65 years and compared them with the reference values derived from a younger general adult population. 


\section{Methods}

\subsection{Subjects}

A cross-sectional descriptive study was carried out on a representative sample of 600 people (257

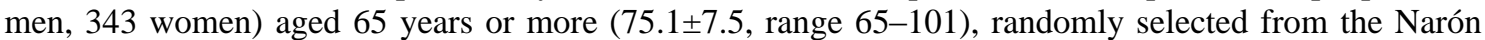
municipal register (A Coruña, Spain). The level of confidence was $95 \%$, accuracy $\pm 4 \%$, and estimation for data losses $10 \%$.

Participants were individually assessed in a health center. Before data collection, all participants were informed about the study and signed the corresponding informed consent form. The study protocol was approved by the Ethics Committee at the University of A Coruña and conformed to the principles embodied in the Declaration of Helsinki.

\subsection{Diabetes and dyslipidemia}

Medical histories were given by the patients or their relatives and their medical records were consulted. The Older Americans Resources and Services (OARS) standardized questionnaire [15] was used to elicit information regarding pathologies and any visits to the doctor within the last 6 months.

Locally derived and used reference intervals were used for evaluation of all the indices (see below). In addition, the World Health Organization (WHO) reference values for diabetes were consulted [16], as were values cited in the 'European guidelines on cardiovascular disease' [17] for high total cholesterol and high low-density lipoprotein cholesterol (dyslipidemia).

\subsection{Blood collection}

Distribution patterns of the biochemical and hematological indices commonly analyzed in clinical chemistry were explored. The specific biochemical indices analyzed were glucose, urea, creatinine, uric acid, aspartate aminotransferase (AST), alanine aminotransferase (ALAT), gamma glutamyl transpeptidase (GGT), total cholesterol, triglycerides, high-density lipoprotein cholesterol (HDLcholesterol), low-density lipoprotein cholesterol (LDL-cholesterol), phosphorus, calcium and thyroidstimulating hormone (TSH). The specific hematological indices analyzed were leucocyte count, erythrocyte count, hemoglobin (Hb), hematocrit (HCT), mean corpuscular volume (MCV), mean corpuscular hemoglobin $(\mathrm{MCH})$, mean corpuscular hemoglobin concentration (MCHC), platelet count and erythrocyte sedimentation rate (ESR).

Blood samples were collected at a primary health care center. For all participants, samples were drawn in the morning, after an overnight fast. Samples for the biochemical indices were collected into SST-gel tubes and those for the hematological indices into EDTA tubes. For determination of the biochemical and hematological indices, analyses were done using 4ml Vacutainer tubes (Becton Dickinson); Seditainer tubes were used for the ESR determination. Biochemistry tubes were centrifugated at room temperature at

$3000 \mathrm{rpm}$. The biochemical indices were determined using an Advia analyzer (Bayer Diagnostics) and the hematological indices were determined using a Beckman-Coulter analyzer. All measurements were reported in SI units.

All analyses were performed in the laboratories of the Complejo Hospitalario Universitario (CHUAC) in A Coruña city on the day of sample collection.

\subsection{Statistical analysis}

A descriptive analysis of the biochemical and hematological indices was carried out; the mean (standard deviation) and median values, maximum and minimum values and the 2.5th and 97.5th percentiles were calculated for each parameter. These values were compared with the CHUAC reference intervals used by the Galician Health Service (SERGAS). Gender-specific intervals were used where appropriate. The number and percentage of sample results that were higher or lower than the CHUAC reference intervals were identified. Results that were more than $5 \%$ above the upper end of the reference interval or $5 \%$ below the lower value were considered to be significantly different from a population perspective.

For any indices on which more than $25 \%$ of participants returned values above those of the reference intervals, further exploration was done. This applied to glucose and cholesterol. The number and percentage of subjects with had glucose levels above the reference interval who reported having been diagnosed with diabetes were noted, as were the number and percentage of subjects with levels of 
cholesterol above the reference value who reported having been diagnosed with dyslipidemia. Finally, a chi-square test was performed to determine whether awareness of a diagnosis of dyslipidemia and diabetes was related to having visited a doctor within the last 6 months.

Statistical analysis was performed using SPPS software version 16.0.2 [18].

\section{Results}

Table 1 summarizes the results for each biochemical and hematological index for the whole sample.

Table 2 shows the reference intervals for the biochemical and hematological indices, and the number and frequency of values from the study sample that fell below and above those intervals.

\subsection{Biochemical indices (see Tables 1 and 2)}

The mean glucose concentration in the study sample was $5.5 \pm 1.4 \mathrm{mmol} / \mathrm{l}$, and $25.0 \%$ of the subjects had glucose values above the CHUAC reference interval. The mean urea concentration was 7.6 \pm 2.5 $\mathrm{mmol} / \mathrm{l}$, and $26.6 \%$ of participants had values above the reference interval. Among women, the mean creatinine level was $89 \pm 18 \mu \mathrm{mol} / \mathrm{l}$ and among men it was $106 \pm 27 \mu \mathrm{mol} / \mathrm{l} ; 13.0 \%$ of the women and $27.2 \%$ of the men had values above the reference interval. The mean concentration of uric acid was $300 \pm 72 \mu \mathrm{mol} / \mathrm{l}$ in women and $377 \pm 90 \mu \mathrm{mol} / \mathrm{l}$ in men; $24.5 \%$ of women and $22.9 \%$ of men had values above the reference interval.

If we refer to transaminases, the mean AST value was $0.37 \pm 0.27 \mu \mathrm{kat} / \mathrm{l}$, and $97 \%$ of participants were within the reference range. The mean ALAT value was $0.43 \pm 0.54 \mu \mathrm{kat} / \mathrm{l}$ in women and $0.45 \pm 0.25 \mu \mathrm{kat} / \mathrm{l}$ in men; $6.3 \%$ of women and $8.1 \%$ of men had ALAT levels above the reference interval. The mean GGT value was $22 \pm 29 \mathrm{U} / \mathrm{l}$ and it was found that $10.1 \%$ of all participants had GGT levels above the reference interval.

As regards cholesterol, mean values were $5.8 \pm 1.1 \mathrm{mmol} / \mathrm{l}$ for total cholesterol, $1.5 \pm 0.4 \mathrm{mmol} / \mathrm{l}$ for HDL-cholesterol and $3.6 \pm 0.9 \mathrm{mmol} / \mathrm{l}$ for LDL-cholesterol; $54.6 \%$ of the sample had levels of total cholesterol above the reference value, and $35.8 \%$ had levels of LDL-cholesterol above the reference value.

The mean concentration of triglycerides was $1.8 \pm 1.1 \mu \mathrm{mol} / \mathrm{l}$, and $12.9 \%$ of participants had levels above the reference interval. The mean concentration of phosphorus was $1.0 \pm 0.2 \mathrm{mmol} / \mathrm{l}$, and $8.6 \%$ of the subjects had levels below the reference interval. The meancalcium concentrationwas $2.3 \pm 0.1 \mathrm{mmol} / \mathrm{l}$, and $99.0 \%$ of participants were within the reference range. The TSH mean value was $2.34 \pm 7.19 \mathrm{mIU} / 1 ; 3.8 \%$ of participants had TSH levels below the reference interval and $2.9 \%$ had levels above.

\subsection{Hematological indices (see Tables 1 and 2)}

The mean sample leucocyte count was $5.0 \pm 2.5 \times 109 / 1$, and $95.1 \%$ of participants fell within the reference interval. The mean erythrocyte count was $4.6 \pm 0.4 \times 1012 / 1$ in women and $4.8 \pm 0.5 \times 1012 / 1$ in men; $22.0 \%$ of women had an erythrocyte above the reference range and $6.2 \%$ below, and $3.5 \%$ of men had a count above the reference range and $15.5 \%$ below. The mean $\mathrm{Hb}$ concentrationwas $8.4 \pm 0.6 \mathrm{mmol} / \mathrm{l}$ inwomenand $9.2 \pm 0.8 \mathrm{mmol} / \mathrm{l}$ inmen; $97.2 \%$ of women and $96.6 \%$ of men had values within the reference interval. The mean HCT value was $0.40 \pm 0.03$ for women and $0.44 \pm 0.04$ for men; $4.8 \%$ of women and $12.6 \%$ of men had HCT values below the reference range, and $3.9 \%$ of women and $3.5 \%$ of men had values above.

The mean MCV within the whole sample was $89 \pm 6 \mathrm{fl}$ and $95.1 \%$ of participants had values within the reference interval. The mean $\mathrm{MCH}$ value was $30.1 \pm 2.1 \mathrm{pg} / \mathrm{cell}$ and $5.3 \%$ of the sample returned values that were above the reference interval. The mean MCHC was $336 \pm 9 \mathrm{~g} / \mathrm{l}$, and $99.5 \%$ of participants returned values within the reference interval. The mean platelet count was $236 \pm 69 \times 109 / 1$, and $95.8 \%$ of participants had counts within the reference interval. The mean ESR was $17 \pm 16 \mathrm{~mm} / \mathrm{h}$, and $24.8 \%$ of participants had counts that were above the reference interval.

\subsection{Glucose and diabetes}

As reported in Table 2, 145 participants had glucose levels above the CHUAC reference range. Table 3 divides this subsample according to whether or not they had had a diagnosis of diabetes; it also similarly reports the diagnosis of diabetes among the 88 participants $(15.2 \%$ of the 580 who had a glucose 
Table 1

Descriptive statistics of laboratory values.

\begin{tabular}{|c|c|c|c|c|c|}
\hline & $n$ & Mean (SD) & Median & 2.5th and 97.5th percentiles & Range \\
\hline \multicolumn{6}{|l|}{ Biochemistry } \\
\hline Glucose (mmol/l) & 580 & $5.5(1.4)$ & 5.2 & $4.0-8.5$ & $2.1-19.3$ \\
\hline \multicolumn{6}{|l|}{ Creatinine $(\mu \mathrm{mol} / \mathrm{l})$} \\
\hline Female & 330 & 89 (18) & 89 & $62-133$ & $62-248$ \\
\hline Female & 326 & $300(72)$ & 294 & $174-474$ & $0-714$ \\
\hline Male & 245 & $377(90)$ & 372 & $222-576$ & $174-810$ \\
\hline $\operatorname{AST}(\mu \mathrm{kat} / \mathrm{l})$ & 574 & $0.37(0.27)$ & 0.35 & $0.22-0.66$ & $0.17-4.33$ \\
\hline \multicolumn{6}{|l|}{$\operatorname{ALAT}(\mu \mathrm{kat} / \mathrm{l})$} \\
\hline Female & 333 & $0.43(0.54)$ & 0.36 & $0.20-0.91$ & $0.13-9.00$ \\
\hline HDL-cholesterol (mmol/l) & 570 & $1.5(0.4)$ & 1.5 & $0.91-2.33$ & $0.6-3.1$ \\
\hline LDL-cholesterol (mmol/1) & 567 & $3.6(0.9)$ & 3.5 & $1.9-5.5$ & $0.9-6.6$ \\
\hline Phosphorus (mmol/l) & 547 & $1.0(0.2)$ & 1.0 & $0.7-1.3$ & $0.6-1.8$ \\
\hline Calcium (mmol/l) & 548 & $2.3(0.1)$ & 2.3 & $2.1-2.5$ & $0.2-2.7$ \\
\hline $\mathrm{TSH}(\mathrm{mIU} / \mathrm{l})$ & 558 & $2.34(7.19)$ & 1.45 & $0.26-5.84$ & $0.00-148.00$ \\
\hline \multicolumn{6}{|l|}{ Hematology } \\
\hline Leucocyte count $\left(\times 10^{9} / 1\right)$ & 549 & $5.0(2.5)$ & 6.3 & $3.9-11.4$ & $2.2-39.9$ \\
\hline \multicolumn{6}{|l|}{ Erythrocyte count $\left(\times 10^{12} / 1\right)$} \\
\hline Female & 331 & $4.6(0.4)$ & 4.6 & $3.7-5.4$ & $3.0-5.9$ \\
\hline Male & 247 & $4.8(0.5)$ & 4.9 & $3.9-5.7$ & $2.0-6.4$ \\
\hline \multicolumn{6}{|l|}{$\mathrm{Hb}(\mathrm{mmol} / \mathrm{l})$} \\
\hline \multicolumn{6}{|l|}{$\mathrm{HCT}$} \\
\hline $\operatorname{MCHC}(g / 1)$ & 578 & $336(9)$ & 336 & $316-355$ & $302-388$ \\
\hline Platelet $\left(\times 10^{9} / 1\right)$ & 578 & $236(69)$ & 230 & $123-401$ & $44-630$ \\
\hline $\operatorname{ESR}(\mathrm{mm} / \mathrm{h})$ & 545 & $17(16)$ & 12 & $2-58$ & $1-120$ \\
\hline
\end{tabular}

$\mathrm{SD}=$ standard deviation; ALAT = alanine aminotransferase; AST = aspartate aminotransferase; ESR = erythrocyte sedimentation rate; GGT=gamma glutamyl transpeptidase;HCT = hematocrit; $\mathrm{Hb}=$ hemoglobin; HDL-cholesterol = high-density lipoprotein cholesterol; LDL-cholesterol = low-density lipoprotein cholesterol; $\mathrm{MCH}=$ mean corpuscular hemoglobin; MCHC=mean corpuscular hemoglobin concentration; $\mathrm{MCV}=$ mean corpuscular volume; $\mathrm{TSH}=$ thyroid-stimulating hormone.

determination) who had glucose values above the WHO reference interval. As can be seen, 51.7\% of people with glucose values above the CHUAC reference interval did not report diabetes. The current WHO diagnostic criteria for diabetes stipulate a concentration of fasting plasma glucose above $7.0 \mathrm{mmol} / \mathrm{l}$ $(126 \mathrm{mg} / \mathrm{dl})$ and 88 participants $(15.2 \%)$ in our sample fulfilled this criterion; of these, $31.8 \%$ had not received a diagnosis of diabetes. Furthermore, the percentage of participants with fasting plasma glucose above $7 \mathrm{mmol} / \mathrm{l}$ who did not report a diabetes diagnosis was significantly higher $(\mathrm{p}=0.001)$ among those who had not visited their doctor within the previous 6 months $(63.2 \%)$ than among those who had visited their doctor (23.2\%).

\subsection{Cholesterol and dyslipidemia}

As reported in Table 2, 317 participants had total cholesterol levels above the CHUAC reference value and 203 had LDL-cholesterol above the CHUAC reference value. Table 4 divides these subsamples according to whether or not they had had a diagnosis of dyslipidemia; it also similarly reports the diagnosis of dyslipidemiaamong the participants who had cholesterol values (total and LDL) above the reference values indicated in the European guidelines [17]. As shown, 72.6\% of subjects with total cholesterol and $72.4 \%$ of those with LDL-cholesterol above the CHUAC reference value did not report having received a diagnosis of dyslipidemia. 
Table 2

Number and percentage of values above and below the reference intervals.

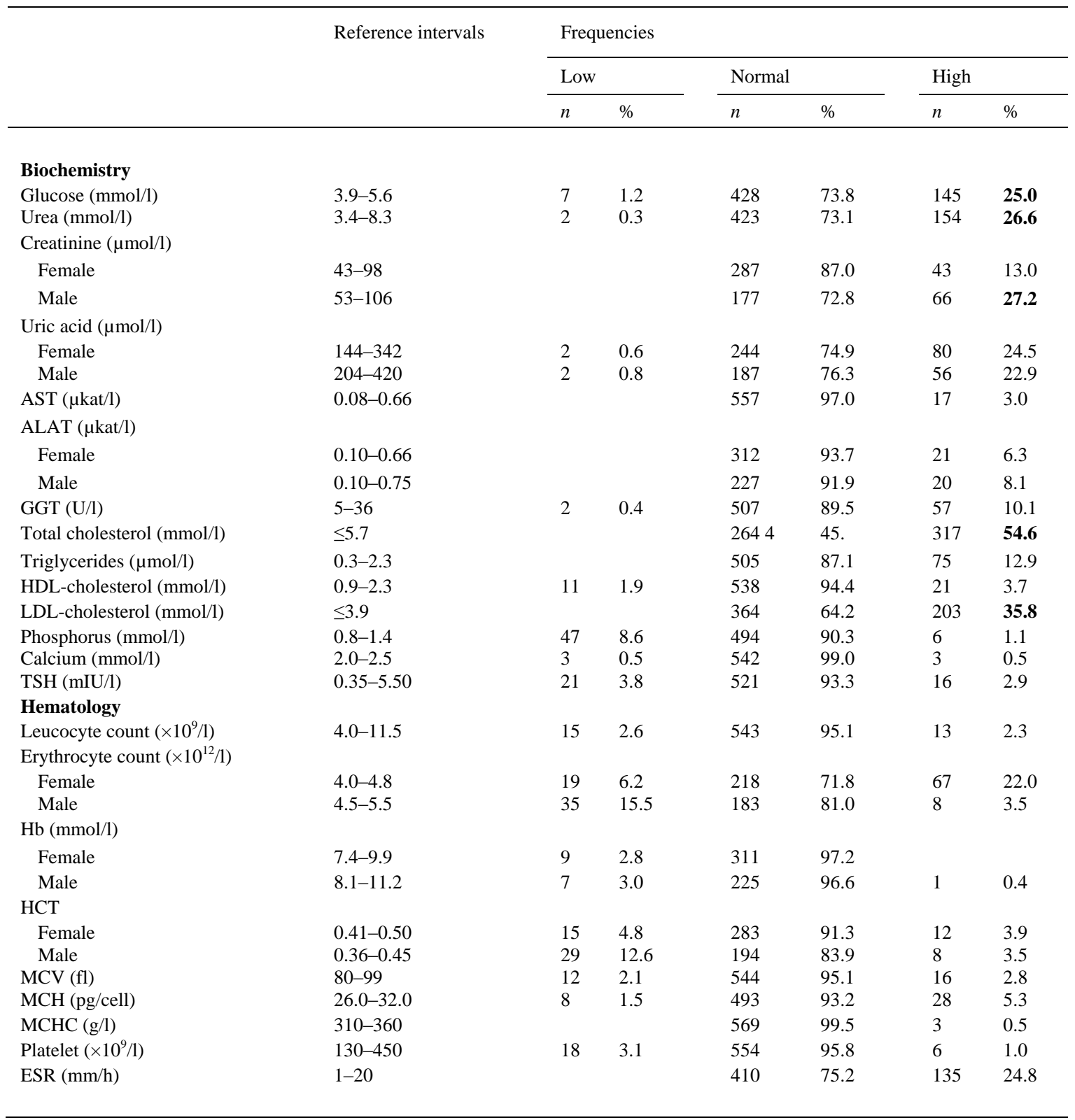

Values in which more than $25 \%$ of participants showed results above those of the reference intervals are presented in bold.

According to the "European guidelines on cardiovascular disease prevention in clinical practice", total plasma cholesterol should be below $5 \mathrm{mmol} / \mathrm{l}$ and LDL-cholesterol should be below $3 \mathrm{mmol} / \mathrm{l}$, but $79.5 \%$ of participants in the present study had cholesterol levels above $5 \mathrm{mmol} / \mathrm{l}$. Of these, $74.9 \%$ did not report having received a diagnosis of dyslipidemia. As regards LDL-cholesterol, $75.5 \%$ of the 567 participants who had a determination had levels above $3 \mathrm{mmol} / \mathrm{l}$. Of these 428 participants, a further $75.5 \%$ did not report having received a diagnosis of dyslipidemia.

In those subjects with a level of total cholesterol above $5 \mathrm{mmol} / \mathrm{l}$, the percentage of those who did not report having received a diagnosis of dyslipidemia was significantly higher $(p=0.001)$ among those who had not visited their doctor within the last 6 months $(88.8 \%)$ than among those who did report a visit (71.6\%). Also, among those subjects with levels of LDL-cholesterol above $3 \mathrm{mmol} / \mathrm{l}$, the percentage of people who did not report having received a diagnosis of dyslipidemia was significantly higher $(p=$ $0.006)$ among those who had not visited their doctor within the last 6 months (87.5\% vs. 12.5\%). 
Table 3

Number and percentage of subjects with glucose levels above the CHUAC and WHO reference intervals reporting a previous diagnosis or no diagnosis of diabetes.

\begin{tabular}{|c|c|c|c|c|}
\hline & \multicolumn{2}{|c|}{ No diagnosis } & \multicolumn{2}{|c|}{ Diagnosis } \\
\hline CHUAC $(\geq 5.6 \mathrm{mmol} / \mathrm{l})$ & 75 & 51.7 & 70 & 48.3 \\
\hline WHO ( $\geq 7.0 \mathrm{mmol} / \mathrm{l})$ & 28 & 31.8 & 60 & 68.2 \\
\hline
\end{tabular}

CHUAC $=$ Complejo Hospitalario Universitario A Coruña; WHO=World Health Organization.

Table 4

Number and percentage of subjects with cholesterol levels above the CHUAC and European reference intervals reporting a previous diagnosis or no diagnosis of dyslipidemia.

\begin{tabular}{|c|c|c|c|c|}
\hline & \multicolumn{2}{|c|}{ No diagnosis } & \multicolumn{2}{|c|}{ Diagnosis } \\
\hline & $n$ & $\%$ & $n$ & $\%$ \\
\hline \multicolumn{5}{|l|}{ CHUAC } \\
\hline Total cholesterol $(\leq 5.7 \mathrm{mmol} / \mathrm{l})$ & 230 & 72.6 & 87 & 27.4 \\
\hline LDL-cholesterol $(\leq 3.9 \mathrm{mmol} / \mathrm{l})$ & 147 & 72.4 & 56 & 27.6 \\
\hline Total cholesterol (>5 mmol/l) & 346 & 74.9 & 116 & 25.1 \\
\hline LDL-cholesterol (>3 mmol/l) & 323 & 75.5 & 105 & 24.5 \\
\hline
\end{tabular}

$\mathrm{CHUAC}=$ Complejo Hospitalario Universitario A Coruña. European reference interval = "European guidelines on cardiovascular disease prevention on clinical practice" [17].

\section{Discussion}

On most of the biochemical indices, a significant percentage of the participants had values outside the reference range. Only in the case of the AST and calcium did more than $95 \%$ of participants have values within the reference intervals. The hematological indices on which more than $95 \%$ of participants were within the reference intervals were leucocyte count, $\mathrm{Hb}, \mathrm{MCV}, \mathrm{MCHC}$ and platelet count. These results are consistent with those reached in a previous study [19]. Thus, few reference intervals for hematological and biochemical indices can be applied directly to community-living elderly subjects without finding an excess of out-of-range values.

In our study, $25 \%$ or more of the participants had levels of glucose, urea, creatinine (in males), total cholesterol and LDL cholesterol above the local reference intervals. Some studies have found that plasma urea levels increase with age, but no relation has been observed between age and plasma creatinine levels $[20,21]$. The high levels of plasma creatinine found in males could be an indication of kidney failure. However, apart from age and sex, the plasma creatinine is also affected by other variables, including weight and ethnicity. Consequently, plasma creatinine levels should not be used alone to assess the kidney function; the Cockroff and Gault formula (CGCC) [22] or the MDRD2 equation [23], which take into account these variables, are more accurate methods of assessment.

The high levels of glucose and cholesterol are particularly noteworthy, as they will have important consequences on health and quality of life, especially for older adults. Diabetes among older adults is a risk factor of cardiovascular events [24] and increases the risk of mortality [25], although the precise role of cholesterol in cardiovascular mortality among the elderly is not as clear as it is for more middle-aged populations. Nevertheless, some studies suggest that total cholesterol is an important risk factor for mortality due to coronary disease in the elderly [26,27].

We found that a large proportion $(31.8 \%)$ of those subjects who fulfilled the WHO's criterion for diabetes (i.e. a glucose level $\geq 7.0 \mathrm{mmol} / \mathrm{l}$ ) did not report having received a diagnosis of diabetes; similarly, large proportions (around three-quarters) of the participants with high levels of total cholesterol and LDL-cholesterol did not report having received a diagnosis of dyslipidemia (Table 4). This is particularly worrying because it indicates these subjects are not aware of their condition and are not in receipt of appropriate medical treatment.

The percentage of people who did not know they fulfilled the diagnostic criteria for these two disorders was significantly higher among those who had not visited the doctor within the last 6 months. In 
other studies it has been shown that having two or more visits to the doctor annually is associated with awareness of dyslipidemia and diabetes [8].

A problem we face when it comes to comparing laboratory values reported in the literature is the use of different techniques and reference values. It would be desirable to harmonize the methods of measurement and the reference values used by different laboratories, as this would mean that patient results would be transferable, which in turn would amplify health benefits and reduce the demand on health systems [28].

Clinical chemistry reference values should be separately specified for elderly persons and these should be re-evaluated regularly to ensure that they remain appropriate [5]. For many of the biochemical and hematological indices we investigated, an excess of values was found outside the reference range set for a younger adult population. It is important to establish optimal reference values for the elderly which take into account the particularities of this group, as this will help the doctors to provide an early and correct diagnosis. In this regard, since laboratory tests are frequently used to detect severe and/or chronic pathologies, the use of adapted, more appropriate reference values for the elderly would help to establish the point at which medical intervention is appropriate for older patients.

\section{Contributors}

We declare that Millán-Calenti designed the study, interpreted the data, and critically reviewed the final version of the manuscript. Sánchez undertook the statistical analysis and wrote the first draft of the manuscript. Lorenzo-López and Maseda managed the literature searches. All authors contributed to and have approved the final version of the manuscript.

\section{Competing interest}

None.

\section{Funding}

Galician Health Service (SERGAS, Consellería de Sanidad y Servicios Sociales) provided us with the facilities and participated as sponsor in the study, but it had not further role in the study design; in the interpretation of data; in the writing the paper; and in the decision to submit the paper for publication.

\section{Acknowledgement}

The authors sincerely thank the Narón Council for providing them access to their elderly people.

\section{References}

[1] Brightwell RF, Crawford GP, Cale JB, Pedler PJ, Bittles AH. Ageing and the haematological profiles of an Australian community. Ann Hum Biol 1998;25:1-10.

[2] Tsang CW, Lazarus R, Smith W, Mitchell P, Koutts J, Burnett L. Hematological indices in an older population sample: derivation of healthy reference values. Clin Chem 1998;44:96-101.

[3] Boulat O, Krieg MA, Janin B, Burckhardt P, Francioli P, Bachmann C. Clinical chemistry variables in normal elderly and healthy ambulatory populations: comparison with reference values. Clin Chim Acta 1998;272:127-35.

[4] Huber KR, Mostafaie N, Stangl G, et al. Clinical chemistry reference values for 75-year-old apparently healthy persons. Clin Chem LabMed2006;44:1355-60.

[5] Carlsson L, Lind L, Larsson A. Reference values for 27 clinical chemistry tests in 70-year-old males and females. Gerontology 2008;56:259-65

[6] Tietz NW, Shuey DF, Wekstein DR. Laboratory values in fit aging individuals sexagenarians through centenarians. Clin Chem 1992;38:1167-85.

[7] Bourdel-Marchasson I, Laksir H, Puget E. Interpreting routine biochemistry in those aged over 65 years: a time for change. Maturitas 2010;66:39-45.

[8] McDonald M, Hertz RP, Unger AN, Lustik MB. Prevalence, awareness, and management of hypertension, dyslipidemia, and diabetes among United States adults aged 65 and older. J Gerontol A Biol Sci Med Sci 2009;64A:256-63.

[9] Plassman BL, Langa KM, Fisher GG, et al. Prevalence of dementia in the United States: the aging, demographics, and memory study. Neuroepidemiology 2007;29:125-32.

[10] Patel KV. Epidemiology of anemia in older adults. Semin Hematol 2008;45:210-7.

[11] Zhang QL, Koenig W, Raum E, Stegmaier C, Brenner H, Rothenbacher D. Epidemiology of chronic kidney disease: results from a population of older adults in Germany. Prev Med 2009;48:122-7.

[12] Marengoni A, Winblad B, Karp A, Fratiglioni L. Prevalence of chronic diseases and multimorbidity among the elderly population in Sweden. Am J Public Health 2008;98:1198-200. 
[13] Nobili A, Franchi C, Pasina L, et al. Drug utilization and polypharmacy in an Italian elderly population: the EPIFARM-elderly project. Pharmacoepidemiol Drug Saf 2011;20:488-96.

[14] Millán-Calenti JC, Tubío J, Pita-Fernández S, et al. Prevalence of functional disability in activities of daily living, instrumental activities of daily living and associated factors, as predictors of morbidity and mortality. Arch Gerontol Geriatr 2010;50:30610.

[15] Fillenbaum GG. Multidimensional functional assessment of older adults. The Duke Older Americans Resources and Services Procedures. Hillsdale, NJ: Lawrence Erlbaum Associates; 1988.

[16] World Health Organization. Definition, diagnosis and classification of diabetes mellitus and its complications. Report of a WHO Consultation. Part 1: Diagnosis and Classification of Diabetes Mellitus. Geneva: World Health Organization; 1999.

[17] De Backer G, Ambrosioni E, Borch-Johnsen K, et al. European guidelines on cardiovascular disease prevention in clinical practice. Third joint task force of European and other societies on cardiovascular disease prevention in clinical practice. Eur Heart J 2003;24:1601-10.

[18] SPSS Inc. SPSS base 16.0.1 for windows user's guide. Chicago: SPSS; 2007.

[19] Janu MR, Creasey H, Grayson DA, et al. Laboratory results in the elderly: the Sydney older persons study. Ann Clin Biochem 2003;40:274-9.

[20] Musch W, Verfaillie L, Decaux G. Age-related increase in plasma urea level and decrease in fractional urea excretion: clinical application in the syndrome of inappropriate secretion of antidiuretic hormone. Clin J Am Soc Nephrol 2006;1:909-14.

[21] Fehrman-Ekholm I, Skeppholm L. Renal function in the elderly ( $>70$ years old) measured by means of iohexol clearance, serum creatinine, serum urea and estimated clearance. Scand J Urol Nephrol 2004;38:73-7.

[22] Cockcroft DW, Gault MH. Prediction of creatinine clearance from serum creatinine. Nephron 1976;16:31-41.

[23] Levey AS, Bosch JP, Lewis JB, Greene T, Rogers N, Roth D. A more accurate method to estimate glomerular filtration rate from serum creatinine: a new prediction equation. Modification of diet in renal disease study group. Ann Intern Med 1999;130:461-70.

[24] Noto D, Cefalù AB, Barbagallo CM, et al. Hypertension and diabetes mellitus are associated with cardiovascular events in the elderly without cardiovascular disease. Results of a 15-year follow-up in a Mediterranean population. Nutr Metab Cardiovasc Dis 2009;19:321-6.

[25] Kronmal RA, Barzilay JI, Smith NL, et al. Mortality in pharmacologically treated older adults with diabetes: the Cardiovascular Health Study, 1989-2001. PLoS Med 2006;3:e400.

[26] Casiglia E, Mazza A, Tikhonoff V, Scarpa R, Schiavon L, Pessina AC. Total cholesterol and mortality in the elderly. J Intern Med 2003;254:353-62.

[27] Corti MC, Guralnik JM, Salive ME, et al. Clarifying the direct relation between total cholesterol levels and death from coronary heart disease in older persons. Ann Intern Med 1997;126:753-60.

[28] Guidi GC, Lippi G, Solero GP, Poli G, Plebani M. Managing transferability of laboratory data. Clin Chim Acta 2006;374:5762. 